\title{
Note sur quelques Reptiles provenant de la Nouvelle-Guinée
}

PAIR

\section{Jean ROUX}

Muséum de Bâle.

La collection erpétologique du Muséum d'Histoire Naturelle de Bâle s'est enrichie dernièrement de plusieurs espèces de Reptiles (Serpents, Lézards, Tortue) collectionnées dans la région de Merauke (Nouvelle-Guinée hollandaise, partie sud) par M. Paul Wirz. L'une d'entre elles est nouvelle pour la science et se trouve décrite ci-dessous.

Voici, accompagnée de quelques notes, la liste des espèces qui ont été identifiées.

\section{Ophidia}

\section{Liasis albertisi Ptrs. et Dor. 1 Ex.}

La longueur totale de cet individu est de $1 \mathrm{~m}$. 80 .

En arrière de l'œil se trouvent seulement 2 postoculaires; 13 sus-labiales, les $5^{\mathrm{e}}, 6^{\mathrm{e}}, 7^{\mathrm{e}}$ bordant l'œil. Les rangées d'écailles autour du corps sont au nombre de 51 . On compte 267 ventrales et 65 sous-caudales dont quelques-unes, ici et là, ne sont pas divisées (en tout 9).

2. Dendrophis calligaster Gthr. 1 Ex.

3. Tropidonotus mairi Gray. 3 Ex.

Chez deux des exemplaires, on ne remarque qu'une seule préoculaire. Sus-labiales au nombre de 8 ; les $3^{e}, 4^{\mathrm{e}}, 5^{\mathrm{e}}$ bordant l'œil. 
4. Fordonia leucobalia (Schleg.). 1 Ex.

Les écailles sont disposées en 25 rangées autour du corps; on compte 147 ventrales et 29 sous-caudales. Aucune plaque ventrale n'est divisée.

5. Myron richardsoni Gray. 1 Ex.

L'internasale est simple. Sus-labiales au nombre de 9 ; la $4^{\mathrm{c}}$ borde l'œil. 3 sous-labiales en contact avec la $1^{\mathrm{e}}$ mentonnière. Plaques ventrales au nombre de 138, sous-caudales 32 .

6. Hydrus platurus (L.). 1 Ex.

7. Pseudechis australis (Gray). 2 Ex.

Chez l'un des exemplaires l'on compte 192 plaques ventrales et 50 sous-caudales; ces dernières sont toutes simples sauf les deux terminales qui sont divisées. L'autre exemplaire possède 190 plaques ventrales et 48 sous-caudales dont aucune n'est divisée.

Chez cet individu on remarque une anomalie dans la grandeur de la $3^{\text {e }}$ sus-labiale, de chaque còté. Cette plaque, au lieu d'être plus grande que la suivante et d'arriver jusqu'au bord inférieur de l'œil est beaucoup plus petite et ne touche pas l'orbite, car il existe deux plaques préoculaires au lieu d'une et l'inférieure s'est formée aux dépens d'une partie de la $3^{\mathrm{e}}$ suslabiale. Le mème fait s'observe aussi chez l'espèce suivante.

8. Pseudechis papuanus Ptrs. et Dor. 1 Ex.

Ce magnifique spécimen a une longueur totale de 2 mètres, dont $1 \mathrm{~m} .75$ pour la tête et le corps et 25 centimètres pour la queue.

Les plaques ventrales sont au nombre de 225; on compte 61 sous-caudales dont une trentaine sont simples.

Chez les exemplaires normaux les sus-labiales sont au nombre de 6 ; la $3^{\mathrm{e}}$ est plus grande que la $4^{\mathrm{e}}$ et ces deux plaques bordent la partie inférieure de l'œil ; en outre, il n'existe qu'une plaque préoculaire. Ce spécimen présente à cet égard une disposition anormale. Du còté gauche, on compte bien 6 labiales supérieures, mais la $3^{\text {e }}$ est réduite à une très petite plaque située sur le bord libre de la mâchoire supérieure et enchâssée entre la $2^{\mathrm{e}}$ et la $4^{\mathrm{e}}$, elle reste donc séparée de l'œil avec lequel 
la $4^{e}$ est seule en contact. La place occupée normalement par la $3^{\mathrm{e}}$ sus-labiale est prise par une grande plaque préoculaire située au-dessous de la normale. Du còté gauche on ne compte que 5 labiales, la $3^{\text {e }}$ ayant complètement disparu et étant remplacée par une grande préoculaire qui s'appuie à la préoculaire normale et s'enfonce en coin entre la $2^{\mathrm{e}}$ et la $4^{\mathrm{e}}$ sus-labiale.

9. Acanthophis antarcticus (Shaw). 3 Ex.

\section{SAURIA.}

10. Lialis burtoni Gray. 2 Ex.

11. Physignathus temporalis (Gthr.). $1 \mathrm{Ex}$.

12. Varanus indicus (Daud.). 2 Ex. juv.

13. Varanus gouldi (Gray). 2 Ex. juv.

14. Varanus prasinus (Schleg.). 1 Ex. juv.

15. Tiliqua gigas (Schn.). 2 Ex.

16. Lygosoma (Hinulia) melanopogon (D. B.). 5 Ex.

17. Lygosoma (Emoa) cyanogaster (Less.). 4 Ex.

18. Lygosoma (Homolepida) wirzi n. sp. 1 Ex.

Cette nouvelle espèce est voisine de Lygosoma crassicauda A. D. qui habite le Queensland, les îles du détroit de Torrès et la région sud de la Nouvelle-Guinée ; elle s'en distingue par le nombre plus élevé des séries d'écailles autour du corps (28 au lieu de 22 ) et par sa coloration. En voici la diagnose :

Corps allongé; membres courts, ne se touchant pas quand on les allonge l'un vers l'autre sur le còté du corps. Distance entre le bout du museau et le membre antérieur contenue $1^{3 / 4}$ fois dans la distance séparant le membre antérieur du membre postérieur. Museau conique, paupière inférieure écailleuse. Narine percée dans une seule plaque nasale; pas de supranasale. Fronto-nasale plus large que longue, formant une longue suture avec la rostrale et une suture plus courte avec la frontale. Frontale plus courte qu'une fronto-pariétale et l'interpariétale prises ensemble, en contact avec les deux premiẹres supraoculaires; 4 supraoculaires dont la deuxième est 
la plus grande. Supraciliaires au nombre de 6 ou 7. 2 plaques fronto-pariétales, la longueur d'une de ces plaques égale à celle de l'interpariétale. Plaques pariétales bien développées, formant une suture en arriêre de l'interpariétale. 3 paires de plaques nuchales. La $5^{\mathrm{e}}$ supra-labiale au-dessous du centre de l'œil. Ouverture auriculaire ronde, assez grande, beaucoup plus grande que la narine.

Ecailles du corps lisses, disposées en 28 rangées, les dorsales plus larges que leurs voisines. Une paire de préanales, plus grandes que les ventrales. La longueur du membre postérieur est égale à la distance séparant le bout du museau de la base du membre antérieur. Le $4^{\mathrm{e}}$ orteil plus long que le $3^{\mathrm{e}}$, portant à sa face inférieure 18 lamelles lisses. Queue épaisse à sa base, $1^{1 / 3}$ fois aussi longue que la tête et le tronc.

Dessus de la tête et du corps brun, les plaques antérieures de la tête non tachées de foncé. Ecailles du dos, des flancs et de la queue tachées de brun noir; ces taches, n'intéressant qu'une écaille, sont peu nombreuses et disposées plus ou moins irrégulièrement. Les flancs sont d'un brun grisâtre, plus clair que le dos, et les écailles sont pour la plupart tachées de brun. Supra-labiales avec sutures tachées de brun. Face inférieure immaculée, d'un blanc grisâtre. Les membres sont de couleur brune, avec taches foncées.

Dimensions de l'exemplaire type $\left(\sigma^{x}\right)$.

Longueur totale.... $175^{\mathrm{mm}}$ Longueur du membre ant.. $15^{\mathrm{mm}}$ Longueur de la tête $12^{\mathrm{mm}}$ Longueur du membre post. $14^{\mathrm{mm}}$ Largeur de la tête. $9^{\mathrm{mim}}$ Longueur de la queue...... 100 $10 \mathrm{~mm}^{\mathrm{mm}}$ Longueur du tronc $63^{\mathrm{mm}}$

\section{ili. Chelonia.}

19. Chelodina novae-guineae Blgr. 1 Ex.

Chez cet individu, la carapace a une longueur de $120^{\mathrm{mm}}$ et une largeur maximum de $83^{\mathrm{mm}}$.

La forme générale de la carapace est ovoïde, la partie la plus large située en arrière; elle n'est que légèrement bombée. La 
plaque nuchale est presque aussi large que longue. La $1^{\mathrm{e}}$ vertébrale est la plus large de la série; les $2^{\mathrm{e}}, 3^{\mathrm{e}}$ et $4^{\mathrm{e}}$ sont plus larges que longues; la $1^{\mathrm{e}}$ est plus large que les costales, la $2^{\mathrm{e}}$ de largeur égale à ces dernières, les autres vertébrales moins larges. La $5^{\mathrm{e}}$ vertébrale est plus longue que large. Les plaques sont pourvues de stries qui convergent plus ou moins vers le centre. Le plastron est beaucoup moins large que la carapace, le lobe postérieur un peu plus étroit que l'antérieur. La plaque intergulaire est assez grande; d'après les indications des auteurs, on voit que ses dimensions, par rapport aux plaques voisines, varient avec l'âge des individus. Chez notre exemplaire elle a une longueur égale environ au double de la suture entre les pectorales, elle est un peu plus longue qu'une plaque pectorale. La suture entre les abdominales et celle entre les fémorales sont sensiblement d'égale longueur, elles sont plus courtes que la suture entre les pectorales. C'est la suture entre les plaques anales qui est la plus grande de toutes.

La tête est de largeur moyenne; la peau recouvrant la partie postérieure du crâne est divisée en petites plaques aplaties qui deviennent bombées sur la nuque et le cou. Membre antérieur avec une série de 5 lamelles transversales à la face supérieure.

La couleur de la carapace est d'un brun noir, celle du plastron d'un brun verdâtre. 


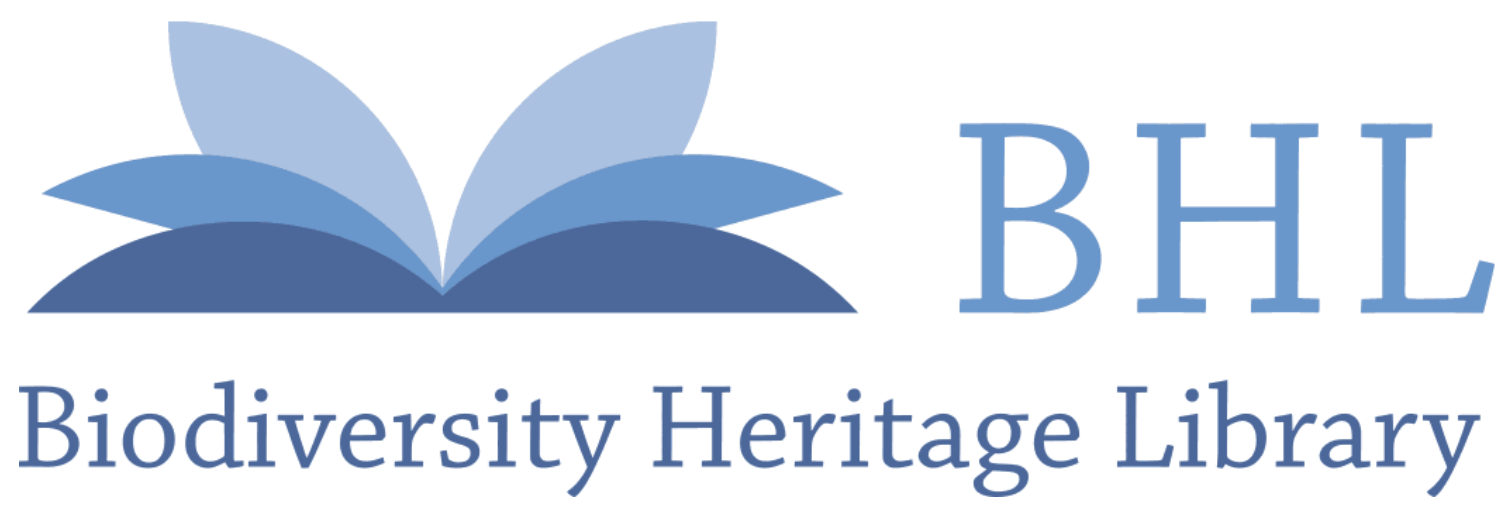

Roux, J. 1919. "Note sur quelques reptiles provenant de la Nouvelle-Guinée." Revue suisse de zoologie 27, 347-351. https://doi.org/10.5962/bhl.part.36328.

View This Item Online: https://www.biodiversitylibrary.org/item/40669

DOI: https://doi.org/10.5962/bhl.part.36328

Permalink: https://www.biodiversitylibrary.org/partpdf/36328

\section{Holding Institution}

MBLWHOI Library

Sponsored by

MBLWHOI Library

\section{Copyright \& Reuse}

Copyright Status: NOT_IN_COPYRIGHT

This document was created from content at the Biodiversity Heritage Library, the world's largest open access digital library for biodiversity literature and archives. Visit BHL at https://www.biodiversitylibrary.org. 